\title{
Leitthema
}

Gefässchirurgie 2015 $\cdot 20: 564-570$

DOI 10.1007/s00772-015-0093-7

Online publiziert: 23. Oktober 2015

(c) Springer-Verlag Berlin Heidelberg 2015

CrossMark

\author{
C. Geppert ${ }^{1} \cdot$ M.K. Widmer ${ }^{1} \cdot$ R. Saxena ${ }^{2} \cdot$ M. Gallieni ${ }^{3}$ \\ ${ }^{1}$ Universitätsklinik für Herz- und Gefässchirurgie, Universität Bern, Bern, Schweiz \\ ${ }^{2}$ Southwestern Medical Center, University of Texas, Dallas, Texas USA \\ ${ }^{3}$ Nephrology, School of Medicine, University of Milano, Milano, Italien
}

\section{Grundlagen der Peritonealdialyse}

\section{Einführung}

Aktuell sind drei Nierenersatzverfahren (NEV) bei terminalen niereninsuffizienten Patienten möglich: Nierentransplantation, Hämodialyse (HD) und Peritonealdialyse (PD). Aufgrund der steigenden Zahl der dialysepflichtigen Patienten wäre auch eine Zunahme aller Verfahren zu erwarten gewesen. Dies ist jedoch nicht der Fall. Während die weltweite Prävalenz der Hämodialyse zunimmt, gibt es in den letzten 20 Jahren eine stete Abnahme der Peritonealdialyse. In Ländern wie den USA ist die Prävalenz der PD passager unter $10 \%$ gefallen und steigt im Moment wieder an. In Mexiko oder in Hongkong ist sie jedoch die erste Wahl der Dialysemöglichkeiten. Mögliche Gründe hierfür sind sozioökonomische Faktoren oder auch Organisationsprobleme (Heim- vs. Zentrumsdialyse).

Weil die Peritonealdialysekatheter abdominal eingelegt werden und deshalb die Operation oftmals nicht durch Gefäßchirurgen erfolgt, soll die Methode an dieser Stelle für den Shuntchirurgen in einer Übersicht dargestellt werden. Vor allem auch darum, weil es eine einfache, effiziente und kostengünstige Methode der Dialyse ist, welche dem Patienten Verantwortung für ein sauberes Management überträgt und neben einem hohen Komfort auch eine gute Sicherheit bietet.

\section{Grundlagen zur Peritonealdialyse}

Der Austausch von Schadstoffen und Flüssigkeit über das Peritoneum [1] erfolgt mittels Diffusion, Konvektion und Ultrafiltration. Die Effizienz der PD ist abhängig von der Beschaffenheit des Peritoneums. Die peritoneale Membran setzt sich aus einer mesothelialen Schicht und einer darunterliegenden Bindegewebeschicht, die aus Kollagen, Mukopolysacchariden, Blut- und Lymphgefäßen besteht, zusammen [8]. Die größte Barriere für diesen Transport ist das peritoneale Endothel. Der transperitoneale Transport wird mit einem Drei-Poren-Modell beschrieben. Dabei werden die Poren in drei Untergruppen eingeteilt: Ultrakleine, kleine und große Poren [2, 3]. Am häufigsten treten die kleinen Poren (40-60

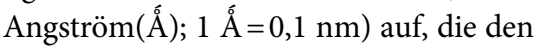
interzellulären Spalten zwischen den endothelialen Zellen entsprechen. Die ultrakleinen Poren (3-5 ̊́), die ebenfalls häufig auftreten, sind auch als transendotheliales Aquaporin-I bekannt [4, 5]. Zusätzlich gibt es die großen Poren (200-300 Á), welche in ihrer Funktion nicht gesichert sind.

Die Diffusion erfolgt durch die kleinen Poren und bildet somit den Hauptteil des Transportmechanismus für kleinmolekulare Substanzen. Die peritoneale Transportfunktion kann mithilfe einer standardisierten Methode (peritonealer Äquilibrium-Test [PET]) untersucht werden. Hierbei wird in $4 \mathrm{~h}$ mit $212,5 \%$ Glukose-Dialysat der Austausch vollzogen [6]. Der Kreatininanteil im Dialysat im Verhältnis zu demjenigen im Plasma nach $4 \mathrm{~h}$ wird als Maßstab für die Einteilung des Peritonealtransportes verwendet. Es gibt vier Kategorien, niedrig, niedrignormal, hoch-normal und hoch. Patienten mit einem hohen Peritonealtransport können den Harnstoff und das Kreatinin rasch ausgleichen und werden ein exzellentes Resultat im Abtransport von kleinen Partikeln haben. Umgekehrt resorbieren die Patienten rasch Glukose aus dem Peritonealraum und verlieren somit den osmotischen Gradienten, was zu einer Verschlechterung der Ultrafiltration führt. Im Gegensatz dazu haben Patienten mit einer geringeren Transportrate niedrigere Harnstoff- und Kreatininclearances und, mindestens zu Beginn, oft eine exzellente Ultrafiltration.

\section{》) Die peritoneale}

Transportfunktion kann

mithilfe des peritonealen

Äquilibrium-Tests [PET]

untersucht werden

Ultrafiltration entsteht, indem mit einer hypertonischen Glukoselösung der kristalloide osmotische Druckgradient zwischen Dialysat und Blut erhöht wird [7]. Die Glukosekonzentration ist zu Beginn der Dialyse am höchsten, nimmt im Verlauf aber ab, da die Glukose ins Blut diffundiert. Infolgedessen ist die Ultrafiltrationsrate initial am schnellsten und nimmt im Verlaufe einer Dialyse ab. Eine unterschiedliche Menge von Glukose wird somit während der PD resorbiert, je nach Verweilzeit des Dialysats, der Transportcharakteristik des Pertitoneums und der Glukosekonzentration.

Zusätzlich zur Diffusion und Ultrafiltration wird die peritoneale Flüssigkeit konstant mit einer Rate von 1-1,5 ml/min resorbiert; entweder direkt über die subdiaphragmalen Lymphgefäße oder über das Interstitium und dann sekundär über die Lymphgefäße und postkapillären Venolen [7].

\section{Modalitäten der Peritonealdialyse}

Die PD erfolgt über einen transkutan eingelegten Abdominalkatheter, der die Instillation von Dialysat in den Peritoneal- 


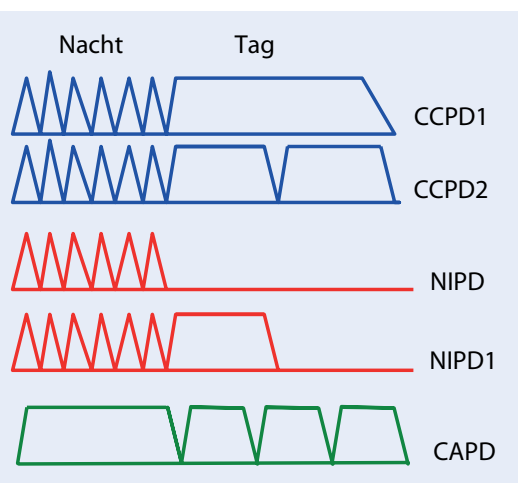

Abb. $1 \Delta$ Bei der Peritonealdialyse sind verschiedene Modalitäten möglich, wann die PDLösungen gewechselt werden
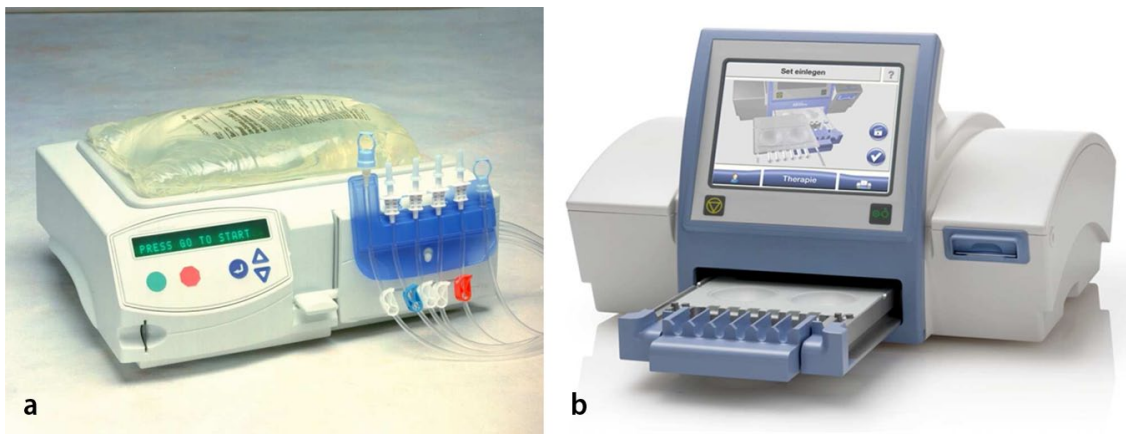

Abb. $2 \Delta$ a, b Die Industrie hat verschieden Modelle von Cyclern entwickelt. a Mit freundlicher Genehmigung von Baxter, Italien. b Mit freundlicher Genehmigung von Fresenius Medical Care Deutschland $\mathrm{GmbH}$

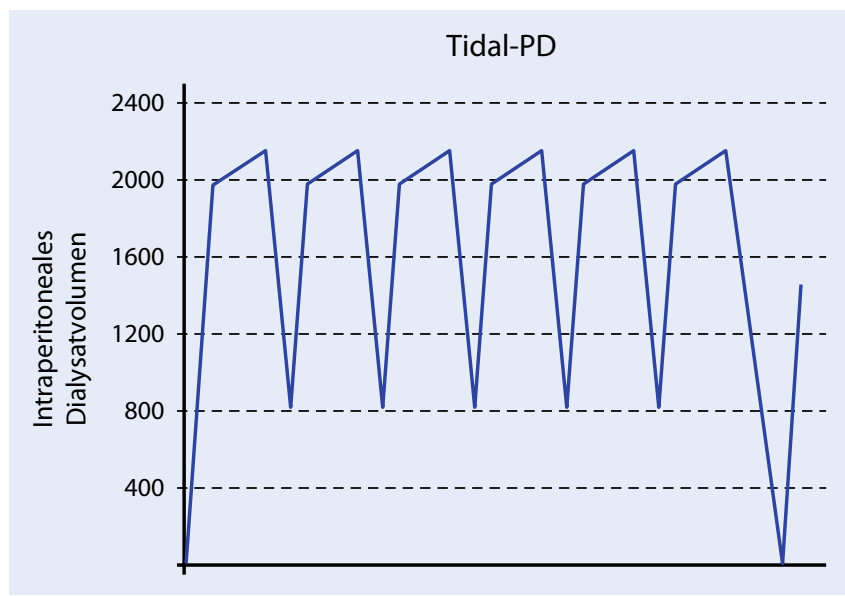

Abb. $3<$ Bei derTital-PD wird nicht das gesamte Dialysat entleert, bevor neue PDLösung instilliert wird raum ermöglicht. Unter normalen Umständen befinden sich 50-100 ml Flüssigkeit im Peritonealraum. Diese Flüssigkeit stammt vor allem aus den Mesothelialzellen. In der PD werden bis zu $3500 \mathrm{ml}$ Dialyselösung in den Peritonealraum instilliert, damit Wasser und Schadstoffe aus den Kapillargefäßen über das Peritoneum ins Dialysat ausgetauscht werden können [8].

Die PD kann manuell erfolgen („,continuous ambulatory peritoneal dialysis“, CAPD) (• Abb. 1) oder mit einem automatischen Antrieb nachts („automated peritoneal dialysis“, APD). Definitionsgemäß erfolgt die CAPD kontinuierlich und kann mit 3-5 Wechseln des Dialysats durchgeführt werden, je nach Restfunktion der Nieren und der Beschaffenheit des Peritoneums. In der APD gibt es die Möglichkeit einer kontinuierlichen Dialyse (intraabdominelle Flüssigkeit $24 \mathrm{~h}$ pro Tag vorhanden). Diese erfolgt als „continuous cycler assisted PD“ (CCPD) oder als intermittierende Dialyse mit nächtli- cher Dialyse und „trockenem“ Abdomen tagsüber („nocturnal intermittent PD“, NIPD) (• Abb. 2). Die NIPD kommt vor allem bei Patienten mit einer Restnierenfunktion zum Tragen.

Die Tidal-APD ist eingeführt worden, um die Effizienz der automatisierten PD zu steigern und um die nächtlichen Alarme bei insuffizienter Drainage zu minimieren. Hierbei wird nicht die gesamte Flüssigkeit gewechselt, sondern nur 40$70 \%$ der instillierten Flüssigkeitsmenge. Dies führt zu schnelleren Austauschzeiten, weniger nächtlichen Störungen und längeren Dialysesitzungen (• Abb. 3).

Die Wahl zwischen APD und CAPD obliegt klinischen und logistischen Faktoren und der Patientenpräferenz. Zum Beispiel ist bei arbeitstätigen Patienten die erste Wahl die APD, da dieses System weniger mit dem Alltag interferiert.

Potenzielle Vorteile der APD sind eine verbesserte Ultrafiltration in Patienten mit hoch-normalen Transportmechanismen, da eine kürzere Austauschzeit eine geringere Glukoseabsorption bedeutet. Dies kommt unter anderem durch einen tieferen intraabdominellen Druck bei liegendem Patienten zustande. Das Risiko von Hernienbildung, Hydrothorax und Katheterleckage ist ebenfalls geringer. Andererseits sind potenzielle Nachteile die höheren Kosten (automatisierter Austauscher, mehr Dialysatmengen und mehr Kathetersets) und die inkomplette Entfernung von großmolekularen Molekülen. Außerdem sind die Patienten „,maschinengebunden“ und es kann keine Dialyse bei einem Automatendefekt erfolgen.

\section{PD-Lösungen}

Herkömmliche PD-Lösungen sind wässrige Lösungen mit Elektrolyten, ähnlich in der Zusammensetzung wie die des Plasmas, einem Bikarbonat-Präkursor (meist Laktat) als Puffer und einem osmotischen Agens, Glukose, für die Ultrafiltration [9]. Unterschiedliche Konzentrationen von Glukose werden verwendet $(1,5 \%, 2,5 \%$, $4,25 \%$ ), um unterschiedliche Osmolalitäten der Flüssigkeiten zu erreichen. Glukose ist generell als osmotischer Agens für die PD weit verbreitet, da kostengünstig und sicher in der Anwendung. Leider ist Glukose rasch resorbiert mit zunehmendem Verlust des osmotischen Gradienten. Außerdem hat dies metabolische Konsequenzen mit Langzeitauswirkungen für den Glukosestoffwechsel bei gewissen $\mathrm{Pa}$ tienten. Die chronische Exposition des Peritoneums mit den Dialyselösungen kann Zytokine aktivieren, die inflammatorische und fibrosierende Veränderungen bewirken und eine Neoangiogenese begünstigen [10]. Dies kann zu Folge haben, dass 
sich bei Langzeit-PD-Patienten der peritoneale Austausch verschlechtert. Aktuell werden zwei Dialysatlösungen angeboten, die nicht auf Glukose basieren: eine icodextrinbasierte Lösung (ein großes Glukosepolymer mit einem Molekulargewicht von $16 \mathrm{kDa}$, was eine bessere Ultrafiltration bei längerer Verweildauer bedeutet, aber schlechter bioverträglich ist bei einem $\mathrm{pH}$-Wert um 5) und eine aminosäurebasierte Lösung mit einer Kombination aus essenziellen und nicht essenziellen Aminosäuren als osmotische Agenzien anstelle von Glukose. Ein Ansatz zur Verbesserung der Bioverträglichkeit sind Glukose basierte Lösungen mit Bikarbonat anstelle von Laktat und mit einem physiologischen $\mathrm{pH}$ als Puffer. Von diesen neuen PD-Lösungen erhofft man sich weniger Komplikationen im Sinne eines eingeschränkten Peritonealtransportes und in der Folge eine langfristig effizientere PD [10-13].

\section{Indikationen und Kontraindikationen für die PD}

Es gibt multiple Gründe und Faktoren, welche die Wahl für eine PD beeinflussen, diese sind längst nicht alle klinisch nachvollziehbar. Unter anderem spielt die Zugänglichkeit zu Dialysezentren und die Erfahrung des behandelnden Nephrologen mit der PD eine wichtige Rolle, wie auch die Patientenbedürfnisse und die Kostenrückerstattung. Dies erklärt auch das breite Spektrum der PD-Prävalenz weltweit von 0-80\%. Eine Zusammenfassung der Indikationen und Kontraindikationen für eine PD sind in der $\bullet$ Tab. 1 aufgelistet.

Erfolgreiche PD-Zentren weisen allesamt hoch qualifizierte und motivierte Ärzte und Pflegefachpersonal mit mehr als 20 PD-Patienten pro Einheit auf.

\section{Katheterwahl und Implantationstechnik}

Es gibt eine große Auswahl an Silikonund Polyurethankatheter für die PD. Die Implantation kann perkutan, laparoskopisch-assistiert oder offen erfolgen [14], dies ist einerseits je nach Modell des Katheters, andererseits von der Expertise des Chirurgen oder des implantieren-

Gefässchirurgie 2015 · 20:564-570 DOI 10.1007/s00772-015-0093-7

(c) Springer-Verlag Berlin Heidelberg 2015

\section{Geppert · M.K. Widmer $\cdot$ R. Saxena $\cdot$ M. Gallieni}

Grundlagen der Peritonealdialyse

\section{Zusammenfassung}

Hintergrund. Neben der Hämodialyse und der Nierentransplantation ist die Peritonealdialyse ein weiteres Nierenersatzverfahren, das aktuell in unseren Breitengraden zu selten angewandt wird.

Ziel der Arbeit. Die Gefäßchirurgen sollen mit den Modalitäten dieser Methode als mögliche Alternative zu einem Hämodialyseshunt vertraut werden.

Methode. Der Artikel gibt einen Überblick über die verfügbaren Techniken zur Peritonealdialyse.

Resultate. Der durch Diffusion, Konvektion und Ultrafiltration bewerkstelligte Austausch von harnpflichtigen Substanzen unter Verwendung von heute meist glukosehaltigem Dialysat in verschiedenen Konzentrationen kann manuell oder automatisch, kontinuierlich oder intermittierend, am Tag oder während der Nacht erfolgen. Es gibt die offene, die laparoskopische oder kombinierte Kathe- terimplantation neben interventionellen Verfahren mit ihren jeweiligen Vor- und Nachteilen. Es stehen auch eine breite Palette von Kathetermodellen zur Verfügung, angefangen vom klassischen Tenckhoff ${ }^{\circledR}$-Katheter bis hin zu sich selbst lokalisierenden WolframKathetern. Katheterdislokationen und -infekte sind die häufigsten Komplikationen bei diesem Verfahren.

Schlussfolgerungen. Die Peritonealdialyse ist bezüglich Mortalität und Morbidität einer Hämodialyse ebenbürtig, bietet aber für den Patienten dank kontinuierlicher Dialyse einen großen Komfort und erlaubt eine ortsungebundenere Bewegungsfreiheit.

\section{Schlüsselwörter}

Peritonealdialyse - Nierenersatzverfahren . Kathetermodelle - Katheterimplantation . Dialysate

\section{Basic principles of peritoneal dialysis}

\section{Abstract}

Background. In addition to hemodialysis and renal transplantation, peritoneal dialysis (PD) is an alternative renal replacement therapy that should receive more attention in Europe.

Aim of the work. A vascular surgeon should be familiar with this method as an alternative to hemodialysis.

Method. This article gives a review of the techniques available for peritoneal dialysis. Results. Peritoneal dialysis can be performed manually or automatically, continuously or intermittently, during the day or even at night using diffusion, convection and ultrafiltration to exchange renal toxins by administration of solutions containing different concentrations of dextrose. Catheter implantation includes open, laparoscopic or a combination of both techniques, besides the in- terventional guided placement with their respective advantages and disadvantages. Different catheters ranging from the classical Tenckhoff ${ }^{\circledR}$ model to self-locating models with tungsten are available. Catheter dislocation and infections are the main complications of peritoneal dialysis.

Conclusions. The mortality and morbidity associated with peritoneal dialysis is similar to that of hemodialysis. The patients benefit from this method as the continuous dialysis offers more comfort and a greater freedom of movement.

Keywords

Peritoneal dialysis · Renal replacement

therapy - Catheter models .

Catheter implantation · Dialysates den Interventionalisten abhängig. Es gibt keine klaren Vor- oder Nachteile hinsichtlich der Art der Implantation [15]. Deshalb gilt als allgemeine Empfehlung: Die Implantationstechnik zu wählen, die für den Patienten am sinnvollsten ist, mit der Option auf einfache Korrektureingriffe, falls notwendig. Die laparoskopisch-assistierte Implantation ist kosten- und per- sonalintensiv und oftmals nur in Allgemeinnarkose möglich. Der große Vorteil liegt bei dieser Methode aber darin, dass man den Katheter offen durch eine paramediane Inzision ins Abdomen einbringen und die Position im Douglas-Raum unter Verwendung einer 5er-Optik kontrollieren kann. Nach einer abdichtenden Tabaksbeutelnaht des Peritoneums 


\begin{tabular}{l} 
Tab. 1 Indikationen und Kontraindikationen für eine Peritonealdialyse (PD) \\
\hline Indikationen \\
\hline - Erste Dialysetherapie bei Patienten mit erhaltener Restfunktion der Niere \\
\hline - Mögliche Nierentransplantation in naher Zukunft \\
\hline - Dialysemethode der ersten Wahl bei (Klein-)Kindern \\
\hline - Kardiovaskuläre Instabilität und Herzversagen (mit PD bessere Prognose als an der Hämodialyse) \\
\hline - Schwierige Gefäßverhältnisse \\
\hline - Blutungsneigung \\
\hline - Phobie vor Nadeln \\
\hline - Aktiver Lebenswandel (Arbeit, Reisen und sportliche Aktivitäten sind einfacher mit einem PD- \\
Katheter) \\
\hline Relative Kontraindikationen \\
\hline - Bauchwandhernien (müssen chirurgisch behoben werden) \\
\hline - Vorhandensein eines lleostomas, Kolostomas, Nephrostomas oder lleum conduits \\
\hline - Chronische Rückenschmerzen mit vorbestehender Diskopathie \\
\hline - Schwer limitierende neurologische Erkrankungen \\
\hline - Blindheit \\
\hline - Eingeschränkte Hand und Fingerbeweglichkeit \\
\hline - Bewegungserkrankungen oder schwere Arthrose, welche die Körperpflege verunmöglichen \\
\hline - Mangelnde Hygiene (Pflegefachpersonen können angelernt werden, die PD durchzuführen) \\
\hline - Adipositas \\
\hline Kontraindikationen \\
\hline - Insuffiziente Peritonealfunktion bei Adhäsionsbauch, Morbus Ormond, Tumorerkrankung oder \\
Versagen der Ultrafiltration \\
\hline - Ausgeprägte Kolondivertikulose \\
\hline - Ausgeprägte entzündliche Darmerkrankung \\
\hline - Chronisch obstruktive Pneumopathie \\
\hline - Ventrikuloperitonealer Shunt \\
\hline - Schwerwiegende aktive psychotische Erkrankung \\
\hline
\end{tabular}

wird der von kranial nach kaudal liegende Katheter palisadenartig durch die hintere Rektusscheide, den Musculus rectus und die vordere Rektusscheide ausgeleitet, um ihn am Schluss leicht bogenförmig subkutan unter Verwendung eines Redon-Stachels nach kaudal zu bringen (- Abb. 4). Wird der Katheter nicht unmittelbar gebraucht, kann man das Ende gänzlich subkutan versenken, wobei der Katheter am Ende mit Flüssigkeit gefüllt und mit einer Ligatur verschlossen werden muss, damit er nicht verstopft. Dieses von Moncrief vorgeschlagene Verfahren erlaubt eine optimale Einheilung des Katheters. Erst wenn der Katheter wirklich gebraucht wird, kann man den Schlauch in lokaler Anästhesie wieder an die Oberfläche bringen [16]. Für Korrektureingriffe und bei abdominal voroperierten $\mathrm{Pa}$ tienten ist die laparoskopische Einlage die Methode der Wahl. Sie erlaubt bei kleinen Zugängen das Lösen von Adhäsionen, um einer Verlegung des Katheters entgegenzuwirken [17]. Idealerweise sollte der be- handelnde Chirurg die offene und laparoskopische Technik beherrschen.

Bei der perkutanen Katheterimplantation können die Kosten deutlich gesenkt werden, da sowohl ein Anästhesist wie auch die Operationssaalnutzung gespart werden können.

In jedem Fall ist ein gut funktionierender PD-Katheter von großer Bedeutung, denn dies hat einen direkten Einfluss auf die Inzidenz der möglichen Komplikationen wie Peritonitis, Dislokation des Katheters oder Probleme bei der Handhabung des Katheters. Etwa $30 \%$ aller gescheiterten PDs sind auf ein Versagen des PD-Katheters zurückzuführen [18]. Ein Katheter sollte einen guten Ein- und Auslauf gewährleisten, knickresistent sein und weder dislozieren noch migrieren. Eine gute Bioverträglichkeit ist ebenso wichtig wie eine gute Resistenz gegen Infekte. Zusätzlich zu diesen Faktoren kommt der Bedienerfreundlichkeit, was die Handhabung und die Pflege des Katheters angeht, eine große Bedeutung zu.

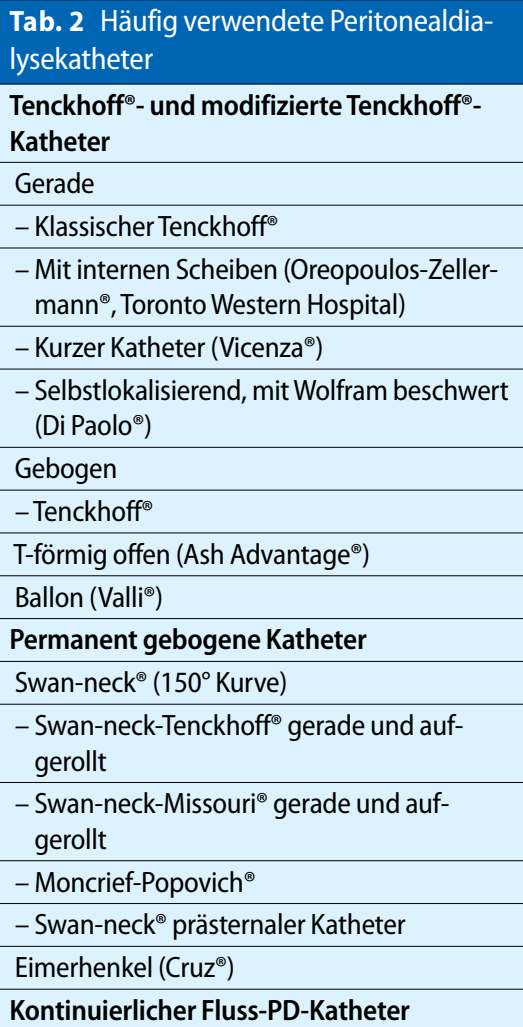

Viele unterschiedliche Kathetertypen sind auf dem Markt erhältlich, die meisten sind jedoch modifizierte Versionen des Original-Tenckhoff ${ }^{\circledR}$-Katheters (• Tab. 2; [19]). Es wird grundsätzlich ein Katheter mit zwei Gewebemuffen, mit einem harmonisch gebogenen und subkutan gelegenen Verlauf und einer kaudal gerichteten Öffnung empfohlen. Um Dialyseleckagen zu vermeiden und eine gute Einheilung des Katheters zu ermöglichen, wird nach der Implantation mindestens eine zweiwöchige Wartezeit bis zur ersten Nutzung empfohlen $[14,15]$. Nur in dringenden Ausnahmefällen soll die PD unmittelbar nach der Implantation begonnen werden, mit kleinen Volumina und nur in liegender Position. Zu den häufigsten Komplikationen zählen Katheterdislokationen, wobei unterschieden wird zwischen Minor- und Majordislokationen. Bei der ersteren verändert sich die Lage der Katheterspitze nur minimal, das heißt, sie bleibt in der unteren Hälfte des Peritonealraums. Bei der Majordislokation migriert die Spitze des Katheters bis in die obere Hälfte des Peritonealraums. Die Katheterdislokation kann Grund für eine Katheterentfernung bzw. gar das Scheitern des Verfahrens sein. Der Hauptgrund für die 


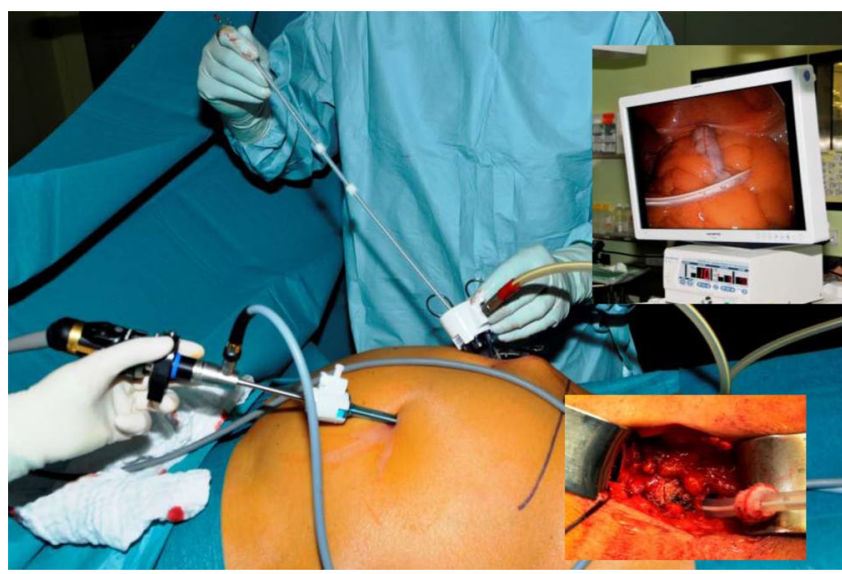

Abb. $4<$ Die laparoskopisch assistierte Kathetereinlage ist übersichtlich und technisch sicher

Dislokation ist die „schwebende “Lage des Katheters auf einer Flüssigkeitskollektion, die wiederum durch die Ein- und Ausfuhr von Dialysatlösung eine „Flut- und EbbeBewegung" simuliert. Diese führt zu kleinen Bewegungen des Katheters, sodass sich dieser im Omentum verfangen kann. Es wurde ein „selbstlokalisierender" Katheter entwickelt, um diese Dislokationen zu vermeiden. Die Spitze ist mit einer Silikonkapsel ausgestattet, die $12 \mathrm{~g}$ Wolfram enthält, das chemisch und physikalisch inert ist. Die beschwerte Spitze verhindert das Schweben der Katheterspitze auf der Flüssigkeit und somit die kleinen Bewegungen, die zur Dislokation führen können [20,21]. Eigene Erfahrungen von einem der Autoren (M.G.) bestätigen, dass sich selbstlokalisierende Katheter gegenüber herkömmlichen Tenckhoff'-Kathetern im Hinblick auf Dislokationen einen Vorteil haben [19]. In einer Multicenterstudie mit 962 Patienten konnte eine signifikante Verbesserung der PD-Rate mit weniger Dislokationen ( 0,8 vs. $22 \%$ ) in der Gruppe mit einem selbstlokalisierenden Katheter gezeigt werden im Vergleich zu den herkömmlichen Tenckhoff'-Kathetern [22]. Die Ausfallquote nach 2 Jahren PD mit selbstlokalisierenden vs. Tenckhoff ${ }^{\circ}$-Kathetern war ebenfalls kleiner in der ersten Gruppe (22 vs 38,4\%). Wir haben aber auch schon eine Umwicklung der Katheterspitze mit Netz gesehen, weil durch die Auftreibung der Katheterspitze diese nicht mehr widerstandslos gleiten kann.

\section{Transferset}

Um vor allem Infektionen zu verhindern, wird die Dialyselösung nie direkt an den Katheter angeschlossen, sondern es wird ein Transferset dazwischen geschaltet. Dies ermöglicht zusätzlich die Kombination der meisten Kathetertypen mit einem jeweils individuellen bzw. herstellerspezifischen Dialysatebeutel. Das Konnektieren des Transfersets an den Katheter bedingt ein steriles Arbeiten (• Abb. 5). Die erste Konnektion erfolgt entweder direkt bei der Implantation des PD-Katheters oder durch das geschulte Personal auf der Dialysestation. Das Transferset wird regelmäßig gewechselt, meist alle 6 Monate. Bevor ein neues Transferset eingesetzt wird, wird der am Katheter befestigte Adaptor über 5 min steril gewaschen.

\section{Komplikationen der PD-Katheter}

Mechanische Komplikationen sind oftmals assoziiert mit einem PD-Katheterversagen [23]. $\mathrm{Zu}$ den Komplikationen zählen die Dysfunktion durch eine intra-/ extraluminale Obstruktion, Fehlplatzierungen, Kathetermigrationen, omentale Verklebungen, Gewebsmuffenmigration, Leckagen, Infektionen und Darmperforationen. Eine Mehrzahl von Peritonitiden können ambulant mit Antibiotika behandelt werden oder es braucht eine chirurgische Intervention bei Tunnelinfekten. Das frühe Erkennen einer möglichen Komplikation ist besonders bei immunkompromittierten Patienten sehr wichtig, um eine Therapie rasch einleiten zu können. Intraluminale Obstruktionen, die durch Koagel oder Fibrinpfropfen verursacht wer-

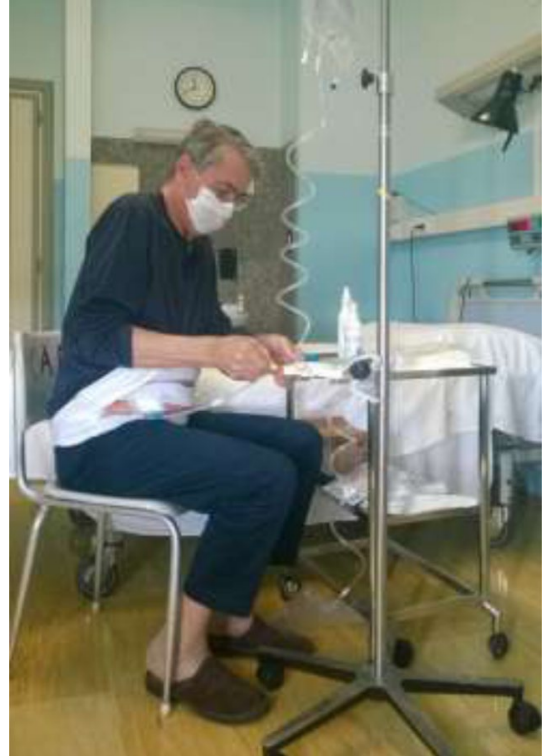

Abb. $5 \Delta$ Patienten müssen geschult werden, unter Einhaltung von Hygienestandards die PDBeutel selbstständig zu wechseln

den, können mit einer Heparinspüllösung oder mit einem gewebespezifischen Plasminogenaktivator behandelt werden.

Katheterassoziierte Komplikationen erhöhen das Risiko für ein Therapieversagen der PD, respektive führen zum Wechsel auf eine Hämodialysebehandlung.

\section{Resultate von PD-Studien}

1995 wurde eine Studie (basierend auf den USRDS-Daten) publiziert, in der ein 19\% höheres Mortalitätsrisiko bei PD-Patienten im Vergleich zu HD-Patienten gezeigt werden konnte [24]. Dies führte zu einem rapiden Rückgang der PD in den USA. Bei Reevaluation der Daten konnte aber, nach Berücksichtigung des Case-Mix, kein signifikant relevanter Unterschied mehr demonstriert werden [25]. In den folgenden Jahren wurde mehrfach gezeigt (basierend auf den Daten aus Kanada, Dänemark und USA), dass das Mortalitätsrisiko bei PD-Patienten signifikant kleiner ist als bei HD-Patienten, außer bei älteren Diabetikerinnen und dies besonders in den ersten 2 bis 3 Jahren nach Dialysebeginn [26-29]. Verschiedene Register und Beobachtungsstudien können keinen klaren Vorteil bzw. Nachteil hinsichtlich des Mortalitätsrisikos für PD- oder HD-Patienten ausmachen. 
Die Inzidenz der Infekte ist ein wichtiger Faktor in den Ergebnis-Studien. Eine 2006 publizierte Beobachtungsstudie, welche die Gesamtinfektrate bei HDund PD-Patienten prospektiv erfasst hatte, fand keinen signifikanten Unterschied in den Kohorten, bis auf die alleinige Inzidenz der Peritonitiden bei den PD-Patienten und die Bakteriämien bei den HDPatienten [30]. Auch eine weitere Studie zeigte keine unterschiedlichen Infektionsraten zwischen erwachsenen Patienten an der Hämodialyse oder PD [31].

\section{) Studien zeigen keine unterschiedlichen Infektionsraten zwischen erwachsenen Patienten an der Hämo- oder Peritonealdialyse}

In den USA haben neue Ergebnisse aus den USRDS-Reports hinsichtlich der Infektrate eine alarmierende Tendenz gezeigt: eine $31 \%$ Zunahme der Infektrate bei dialysepflichtigen Patienten von 1994 bis 2010 [32]. Besonders bei den HD-Patienten ist diese Zunahme in den beiden ersten Jahren nach HD-Beginn im stationären Bereich festzustellen: 1,69 stationäre Behandlungen pro Patientenjahr für die PD-Patienten im Vergleich zu 1,89 für die HD-Patienten. Diese Zunahme wird auf den vermehrten Einsatz von tunnelierten HD-Kathetern zurückgeführt. Umgekehrt ist ein steter Rückgang der Hospitalisationen seit 1993 für die Behandlung von Peritonitiden und von PD-Katheterassoziierten Infekten festzustellen.

\section{Fazit für die Praxis}

- Der Vorbehalt, dass die PD mit höheren Infektraten und mehr Komplikationen einhergeht, ist aufgrund der vorliegenden Daten nicht begründet.

- Weltweit gesehen wird die PD immer noch zu wenig eingesetzt, obschon sie hinsichtlich der klinischen Ergebnisse mindestens gleichwertig, kostengünstiger, einfach und praktikabel ist. Ursächlich dafür sind patientenund arztassoziierte Faktoren wie Unkenntnis, Unwille, psychosoziale und ökonomische Umstände und mangelnde Ausbildung.
- Es braucht ein engagiertes Team von Nephrologen, Pflegepersonal und Chirurgen, um den Service für die PD auf ein qualitativ hoch stehendes Niveau zu heben und dieses auch zu halten.

PD Dr. med. et MME (unibe)
$\begin{aligned} & \text { M.K. Widmer } \\ & \text { Universitätsklinik für Herz- } \\ & \text { und Gefässchirurgie, } \\ & \text { Universität Bern } \\ & 3010 \text { Bern } \\ & \text { matthias.widmer@insel.ch }\end{aligned}$

Danksagung. Wir danken Prof. Dr. med. D.E. Uehlinger, Universitätsklinik für Nerphrologie, Hypertonie und klinische Pharmakologie, Universitätsspital Bern für die kritische Durchsicht des Manuskriptes.

\section{Einhaltung ethischer Richtlinien}

Interessenkonflikt. C. Geppert, M.K. Widmer, R. Saxena und M. Gallieni geben an, dass kein Interessenkonflikt besteht.

Dieser Beitrag beinhaltet keine Studien an Menschen oder Tieren. Alle Patienten, die über Bildmaterial oder anderweitige Angaben innerhalb des Manuskripts zu identifizieren sind, haben hierzu ihre schriftliche Einwilligung gegeben.

\section{Literatur}

1. Leypoldt JK (2002) Solute transport across the peritoneal membrane. J Am Soc Nephrol 13:S84-S91

2. Rippe B, Stelin G, Haraldsson B (1991) Computer simulations of peritoneal fluid transport in CAPD. Kidney Int 40:315-325

3. Flessner MF (1994) Osmotic barrier of the parietal peritoneum. Am J Physiol 267:F861-F870

4. Pannekeet MM, Mulder JB et al (1996) Demonstration of aquaporin-CHIP in peritoneal tissue of uremic and CAPD patients. Perit Dial int 16(Suppl 1):S54-S57

5. Lai KN, Lam MF, Leung JC (2003) Peritoneal function: the role of aquaporins. Perit Dial Int 23(Suppl 2):S20-S25

6. Twardowski ZJ, Nolph KD, Khanna R et al (1987) Peritoneal equilibration test. Perit Dial Bull 7:138147

7. Kreidet RT, Lindholm B, Rippe B (2000) Pathophysiology of peritoneal membrane failure. Perit Dial Int 20(Suppl 4):S22-S42

8. Dobbie JW (1988) From philosopher to fish: comparative anatomy of the peritoneal cavity as an excretory organ and its significance for peritoneal dialysis in man. Perit Dial Int 8:4-8

9. Feriani M (2000) Use of different buffers in peritoneal dialysis. Semin Dialysis 13:256-260

10. Martikainen TA, Teppo A-M, Grönhagen-Riska C, Ekstran AV (2005) Glucose-free dialysis solutions: inductors of inflammation or preservers of peritoneal membrane? Perit Dial Int 25:453-460
11. Ayuzawa N, Ishibashi Y, Takazawa Y, Kume H, Fujita T (2012) Peritoneal morphology after long-term peritoneal dialysis with biocompatible fluid: recent clinical practice in japan. Perit Dial Int 32:159-167

12. le Poole $C Y$, van Ittersum FJ, Valentijn RM et al (2012) „NEPP" peritoneal dialysis regimen has beneficial effects on plasma CEL and 3-DG, but not pentosidine, CML, and MGO. Perit Dial Int 32:4554

13. le Poole CY, Welten AGA, ter Wee PM et al (2012) A peritoneal dialysis regimen low in glucose and glucose degradation products results in increased cancer antigen 125 and peritoneal activation. Perit Dial Int 32:305-315

14. Figueiredo A, Bak-Leong Goh B-L, Jenkins S et al (2010) Clinical practice guidelines for peritoneal access. Perit Dial Int 30:424-429

15. Hwang C, Davidson I, Santarelli S et al (2013) Peritoneal dialysis access: open versus laparoscopic surgical techniques. JVasc Access 14:307-317

16. Moncrief JW, Popovich RP, Broadrick LJ et al (1993) The Moncrief-Popovich catheter. A new peritoneal access technique for patients on peritoneal dialysis. ASAIO J 39(1):62-65

17. Asif A (2004) Peritoneal dialysis access-related procedures by nephrologists. Semin Dial 17:398-406

18. Ash SR (1990) Chronic peritoneal dialysis catheters: effect of catheter design, materials and location. Semin Dial 3:39-46

19. Gallieni M (2012) Characteristics of available peritoneal dialysis catheters. In: Davidson I, Gallieni M, Saxena R (Hrsg) Peritoneal dialysis - surgical technique and medical management. DAVIDSON MEDICAL SERIES, Bd I. Divadi LLC, Dallas

20. Di Paolo N, Petrini G, Garosi G, Buoncristiani U, Berardi S, Monaci G (1996) A new self-locating peritoneal catheter. Perit Dial Int 16:623-627

21. Di Paolo N, Capotondo L, Brardi S, Nicolai G (2010) The self-locating peritoneal catheter: fifteen years of experience. Perit Dial Int 30:504-505

22. Di Paolo N, Capotondo L, Sansoni E et al (2004) The self-locating catheter: clinical experience and follow-up. Perit Dial Int 24:359-364

23. Singh N, Davidson I, Minhajuddin A, Gieser S, Nurenberg M, Saxena R (2010) Risk factors associated with peritoneal dialysis catheter survival: a 9-year single center study in 315 patients. J Vasc Access 11:316-322

24. Bloembergen WE, Port FK, Mauger EA, Wolfe RA (1995) A comparison of mortality between patients treated with hemodialysis and peritoneal dialysis. J Am Soc Nephrol 6:177-183

25. Vonesh EF, Moran J (1999) Mortality in end-stage renal disease: a reassessment of differences between patients treated with hemodialysis and peritoneal dialysis. J Am Soc Nephrol 10:354-365

26. Heaf JG, Lokkegaarg H, Madsen M (2002) Initial survival advantage of peritoneal dialysis relative to hemodialysis. Nephrol Dial Transplant 17:112-117

27. Fenton SSA, Schaubel DE, Desmeules $M$ et al (1997) Hemodialysis versus peritoneal dialysis: a comparison of adjusted mortality rates. Am J Kidney Dis 30:334-342

28. Weinhandl ED, Foley RN, Gilbertson DT, Arneson TJ, Snyder JJ, Collins AJ (2010) Propensity-matched mortality comparison of incident hemodialysis and peritoneal dialysis patients. J Am Soc Nephrol 21:499-506

29. Lukowsky LR, Mehrotra R, Kheifets L, Arah OA, Nissenson AR, Kalantar-Zadeh K (2013) Comparing mortality of peritoneal and hemodialysis patients in the first 2 years of dialysis therapy: a marginal structural model analysis. Clin J Am Soc Nephrol 8:619-628 
30. Aslam N, Bernardini J, Fried F, Burr R, Piraino B (2006) Comparison of infectious complications between incident hemodialysis and peritoneal dialysis patients. Clin J Am Soc Nephrol 1:12261233

31. Williams VR, QuinnR, Callery S, Kiss A, Oliver MJ (2011) The impact of treatment modality on infection-related hospitalization rates in peritoneal dialysis and hemodialysis patients. Perit Dial Int 31:440-449

32. U.S. Renal Data System, USRDS (2013) Annual Data Report: Atlas of Chronic Kidney Disease and EndStage Renal Disease in the United States, National Institutes of Health, National Institute of Diabetes and Digestive and Kidney Diseases, Bethesda, MD, 2013. www.usrds.org. Zugegriffen: 21. Sep. 2014

\section{Neue Impfempfehlungen der STIKO veröffentlicht}

Die STIKO hat ihre Impfempfehlungen aktualisiert. Änderungen gibt es u.a. bei der Pneumokokken-, Meningokokken-B-, Gelbfieber- und Windpockenimpfung. Bei der Pneumokokken-Impfung sollen Säuglinge zukünftig nur drei statt bisher vier Impfstoffdosen eines Pneumokokken-Konjugatimpfstoffs erhalten, im Alter von 2, 4 und 11-14 Monaten (2+1-Impfschema). Für Frühgeborene (Geburt vor der vollendeten 37 . Schwangerschaftswoche) wird unverändert das 3+1-Schema empfohlen. Es gibt geschätzte elf Fälle von Pneumokokken-Infektionen pro 100.000 Kinder im Alter unter fünf Jahren.

Die STIKO empfiehlt zukünftig für Personen mit spezifischen Grundkrankheiten eine Impfung gegen Meningokokken B (Indikationsimpfung).

Bei der Gelbfieberimpfung wird keine Auffrischimpfung mehr empfohlen.

Bei fehlender Immunität gegen das VarizellaZoster-Virus, wenn ein erhöhtes Risiko für eine schwere Windpocken-Infektion besteht und nicht mit dem Lebendimpfstoff geimpft werden kann: Hier hat die STIKO ihre Empfehlung erweitert, hinsichtlich des Personenkreises (Frühgeborene mit Kontakt zum Erreger) und des Zeitraums der Anwendung (bis zu 10 Tage nach Erregerkontakt). Zudem wurden die Masern-Impfempfehlungen redaktionell überarbeitet.

\section{Ärzte erwarten Aufnahme von Apps in die Leitlinien}

43,8\% der Ärzte in Deutschland erwarten, dass therapieunterstützende Apps innerhalb der nächsten zehn Jahre in die Leitlinien aufgenommen werden. Zu diesem Ergebnis kommt die aktuelle eHealth-Studie 2015 aus der Studienreihe "Ärzte im Zukunftsmarkt Gesundheit" der Stiftung Gesundheit. Noch im Vorjahr hatten mehr als zwei Drittel der befragten Ärzte bezweifelt, dass Apps in Zukunft überhaupt den Gesundheitszustand ihrer Patienten überwachen könnten oder sich in dieser Funktion durchsetzen würden. In der Vergangenheit hatten sich Ärzte bei diesem Thema sehr kritisch und zurückhaltend bis ablehnend gezeigt. Die initialen Berührungsängste mit den neuen Medien und Möglichkeiten haben sie offenbar nun aber abgelegt. Dies betrifft nicht nur jüngere Ärzte, bei denen dies thematisch bedingt zu erwarten gewesen wäre, sondern ganz im Gegenteil: Die Altersstruktur der Ärzte, die sich an der Studie beteiligt haben, entspricht weitgehend den Anteilen in der Gesamtärzteschaft, wobei die Altersgruppen über 51 Jahren sogar überproportional vertreten waren. Die aktuelle Studie sowie alle bisherigen Ausgaben der Studienreihe „Ärzte im Zukunftsmarkt Gesundheit" sind auf der Website der Stiftung Gesundheit zugänglich:

www.stiftung-gesundheit.de/stiftung/ studien.htm www.stiko.de www.rki.de/impfen 